\title{
AN INTEGRATED SPACE PHYSICS INSTRUMENT (ISPI) FOR SOLAR PROBE
}

\author{
B. T. Tsurutani, K. Leschly, \\ S. Nikzad, and E. R. Fossum \\ Jet Propulsion Laboratory \\ 4800 Oak Grove Drive \\ Pasadena, CA 91109 \\ 818-354-7559 \\ G. Murphy \\ Design_Net Engr. LLC \\ 600 W. County Line Road \\ \# 31-202 \\ Highlands Ranch, CO 80126 \\ 303-683-6544
T. L. Killeen and B. C. Kennedy
University of Michigan 2455 Hayward St.
Ann Arbor, MI 48109
313-747-3435

\author{
A. M. Title and D. L. Chenette \\ Lockheed-Martin Palo Alto \\ Research Labs \\ 3251 Hanover St. \\ Palo Alto, CA 94304 \\ 415-424-4034 \\ G. Musmann and F. Gliem \\ Technical University of \\ Braunschweig \\ Braunschweig, Germany \\ 49-531-391-5217 \\ S. L. Moses \\ TRW Space and Technology \\ Group \\ 1 Space Park \\ Redondo Beach, CA 90278 \\ 310-812-0075
}

\author{
I. Mann \\ Max Planck Inst. for Aeronomie \\ Lindau-Katlenburg, D-3411 \\ Germany \\ 49-556-979291
}
A. J. Tuzzolino
University of Chicago
933 E. 56th St.
Chicago, Ill. 60637
312-702-7798

\begin{abstract}
Instruments for the Solar Probe mission must be designed not only to address the unique scientific measurement requirements, but must be compatible with the modest resource dollars as well as tight constraints on mass and power. Another unique aspect of the Solar Probe mission is its constraint on telemetry and the fact that the prime science is conducted in a single flyby. The instrument system must be optimized to take advantage of the telemetry and observing time available. JPL, together with industry and university partners, is designing an Integrated Space Physics Instrument (ISPI) which will measure magnetic fields, plasma waves, thermal plasma, energetic particles, dust, and perform EUV/visible and coronal imaging for the Solar Probe mission. ISPI uses a new architecture and incorporates technology which not only eliminates unnecessary duplication of function, but allows sensors to share data and optimize science. The current ISPI design goal (for a flight package) is a 5 kilogram / 10 watt payload.
\end{abstract}

\section{INTRODUCTION}

Programmatic limitations, technical or environmental constraints, and an exploratory mission like Solar Probe bring design and implementation challenges that require rethinking not just the individual instrument design, but the way NASA designs payloads. To truly save time, dollars, and physical resources, a new methodology in instrument development, test, integration, and operations is needed. Our traditional methods will not achieve the results desired within the resource constraints and the result will be a mission which sacrifices its scientific value.

Likewise, sensors which have a single opportunity to bring new insight into processes that control the origin and acceleration of the solar wind with observation at only 4 solar radii must be flexible, adaptable, and synchronized. Designing instruments which require ground intervention, place costly constraints on the $\mathrm{S} / \mathrm{C}$ design, or which are more suited to refined observation rather than exploration limit the science return of this opportunity.

Two key factors will directly affect the cost, schedule, and scientific merit of the Solar Probe payload. First is the use of an architecture which enables more adaptable science, eases integration and development time (and cost) while increasing reliability. Second is the ability to capitalize on technology which can miniaturize, increase sensor performance and further complex objectives of this mission. The ISPI is a program at JPL dedicated to fostering the incorporation of new technology both into sensors and subsystems of space instruments and to the development of an instrument architecture which allows more efficient use of resources. A version of the ISPI incorporating appropriate sensors has been proposed as a candidate instrument for Solar Probe. 


\section{ISPI ARCHITECTURE}

The ISPI architecture is designed with the following objectives:

- enable reduction of resource requirements by elimination of duplication;

- focus reliability at the subsystem level;

- dramatically simplify the task of integration and test;

- enable scientists (Co-Investigators) to concentrate efforts on the sensor development;

- provide as much flexibility as possible to the $\mathrm{Co}-\mathrm{I}$ in software / firmware development without compromising schedule or reliability;

- allow increased science return by with a "smarter" data compression process, and enable "interactive" operation between sensors. are:

The ISPI Architecture is illustrated in Figure 1. Key features that enable ISPI to meet the the above objectives

1. redundant, hardened, and cross-strapped central data processors and power converters which eliminate the need for each sensor to have powerful DPU and to design their own power converters;

2. "switched" architecture which allows increased throughput at narrower bus widths and slower clock speeds and modular addition of sensors, $\mathrm{I} / \mathrm{F}$ modules, memory, etc.;

3. a single interface to $\mathrm{S} / \mathrm{C}$ data and power subsystems which greatly simplifies integration;

4. pre-processors which are sized and matched to the specific sensor processing tasks and data rates eliminate "overdesign" and excess capacity;

5. standard sensor interfaces (digital) coupled with a testbed development environment allow simple integration of the sensors to the subsystem;

6. a software architecture with "functional separability" for sensor specific pre-processing, interactive science, and generic spacecraft $1 / O$ functions which minimizes duplication of software development and focuses resources on the science processing.

The ISPI architecture requires a re-definition of "instrument". "Instrument" is now the whole science payload (the $\mathrm{S} / \mathrm{C}$ only sees one instrument) and the basic science measurements are performed by "sensor" elements. The definition of "sensor" for ISPI includes the actual in-situ or remote sensing hardware / firmware, associated analog processing and A/D conversion functions, and the sensor communication interface module in the pre-processor. The sensors are mounted remotely from the subsystem electronics and have a simple mechanical / thermal interface to the $\mathrm{S} / \mathrm{C}$ (no direct electrical interface). This provides more flexibility in the mechanical accommodation and thermal control, reduces analysis and testing, and increases reliability. The DPU that is part of the ISPI could easily double as central data processor serving an entire "sciencecraft" if attitude control, communication, and power management modules were added (using the same pre-processor interface as the science sensors). This is appropriate for modest, single PI, MIDEX class missions but is not the implementation planned for solar probe.

\section{Implementation in Hardware/Software}

The architecture uses three interface protocols: external (S/C to ISPI); internal (e.g. DPU to pre-processor); and sensor (i.e.pre-processor to sensor). Once defined functionally, the specific hardware implementation of these interfaces is implemented with off-the-shelf components in the testbed and then packaged with flight quality components in the protoflight units, but the interface protocol (the virtual interface) will remain constant.

The ISPI hardware realiziation of this architecture involves a division of responsibility between the NASA sponsoring institution (e.g. JPL) which builds the ISPI subsystem and testbed, and the sensor Co-Is who build the sensors and design the pre-processor software. Three general types of pre-processors are planned and will be 
available to the Co-I from the ISPI project: the "Wave / Image pre-processor" which is a DSP based engine for handling massive amounts of data and doing compression, transforms, filtering, etc.; the "Event pre-processor" which is a state machine based system oriented toward fast "look-up-table" processing of event oriented data (the event pre-processor serves sensors like energetic particle detectors and dust detectors); and last of all the "low-rate micro controller" preprocessor which handles instruments with lower data rates but lots of analog or digital control functions (these include sensors like magnetometers, RPAs spectrometers, etc.). The location of the pre-processor can either be with the sensor or within the ISPI subsystem. When located at the sensor the external interface "speaks" directly through the switch to the DPU, memory, etc. When located in the subsystem, the interface to the sensor is a standard serial protocol which uses commercially available interface circuits. When located in the sensor, JPL will provide the selected pre-processor hardware, but the Co-I must develop the software.

Dual, cross-strapped, high reliability converters and regulators provide required voltages to each sensor. The central power distribution relieves the sensor developer of yet another responsibility and allows higher efficiency in conversion.

The software has a similar division of function and responsibility. ISPI recognizes three classes of software: generic--software which handles command and control, telemetry, housekeeping, GSE interface, EDAC etc.; sensor specific--which handles reduction of sensor specific data (this is mostly contained in the pre-processor but may have modules contained in the central DPU); and interactive--software which enables sensors to exchange data, or allows "intelligent" configuration.

Modularity is a key element in the serviceability and reliability of the software and the fact that the sensor developer is provided with a testbed environment will enable much more efficient code development without the duplication normally present on a science payload. Since the sensor developer is provided with the hardware preprocessor and the software interface module to ISPI (communication protocol to the DPU) they need only design that software which directly affects sensor operation and data handling.

\section{The ISPI Testbed: Impact on Development and Integration}

Much of the advantage of the ISPI architecture to cost and schedule is realized by the implementation of an ISPI testbed. The testbed serves a purpose broader that just that of interface verification and software development. The testbed is built as part of the ISPI program to provide a laboratory test environment for various elements of the ISPI architecture, technology and sensors. The testbed is developed in stages, mostly with off the shelf components, in a modular fashion: the first stage implements the basic switched architecture, the GSE interface, most of the generic software modules, memory, and peripherals; the second stage incorporates the pre-processors and their sensors or sensor simulators and enables development of various interactive software.

When complete, the testbed exists as part of the ISPI development and is available as a useful tool for mission specific design. Since most of the software and interface protocols remain constant (the S/C interface may change from mission to mission), only sensor specific and interactive software must be developed. This dramatically reduces the cost of preparing for a specific mission. In the early design phase, prior to CDR, the ISPI testbed will have a virtual interface for use by the sensor investigator over the internet. This allows development and test of certain sensor specific software as well as interactive software modules. Later, during phase C/D, the ISPI testbed is packaged as a simulator which is available to sensor designers for further verification and test. Once a sensor has been packaged for flight it is brought to JPL to be electrically interfaced to the flight subsystem package. Since all previous modules have been tested and since the interface hardware is standardized, the integration of the sensors becomes a matter which focuses primarily on the interactive software and the verification of all command and control functions. By the time the ISPI subsystem integration is complete, all that remains is to plug the system into the spacecraft. We now integrate one system with one power and data interface instead of many. 


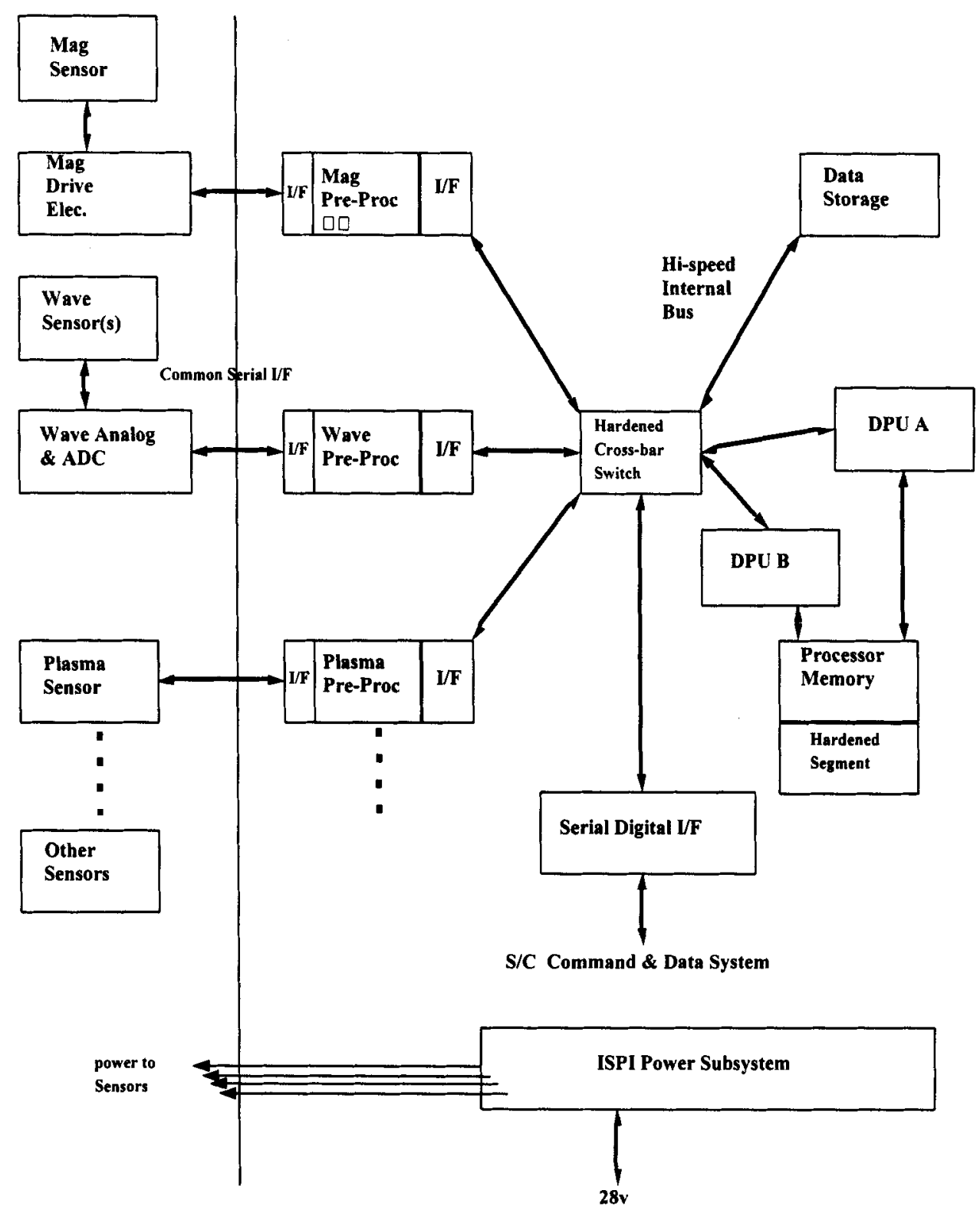

FIGURE 1. ISPI Architecture Allows Functional Partitioning of Hardware and Software.

\section{ISPI TECHNOLOGY}

The ISPI program does not develop technology--it seeks to utilize technology. The ISPI partners each bring a unique technology which is enabling to the architecture, the sensors, or the methodology of the ISPI program. Much of our emphasis is on sensor technology and in particular we select technologies based on their ability to increase sensor performance or enhance the science capability of a mission. The ISPI technology utilization program can be defined as much by what it does not do as what it does do. We do not for example develop a new processor--we will utilize the existing developments from NASA, DoD, and the commercial sectors; we do not concern ourselves much with the packaging technology--when ready for Phase C/D development a particular ISPI configuration will be packaged according to the best compromise between weight / volume and cost; we do not reinvent standard protocols--we utilize existing standards; we do not select a technology just to prove that technology--we select a technology if it can be proven to enhance the capability and reduce the cost of a particular mission. Table 1 lists some of the ISPI advanced technologies selected as candidates for the Solar Probe mission along with a brief synopsis of their advantages. 
TABLE 1. Selected Sample of ISPI Technologies.

\begin{tabular}{|l|l|}
\hline \multicolumn{1}{|c|}{ Technology (source) } & \multicolumn{1}{c|}{ Application or advantage to ISPI } \\
\hline Switched Architecture (commercial) & $\begin{array}{l}\text { Allows efficient data transfer, less contention, less } \\
\text { power, modular }\end{array}$ \\
\hline Operate Through (military) & $\begin{array}{l}\text { Allows robust computing, fault tolerance and recovery, } \\
\text { can be applied to commercial processors }\end{array}$ \\
\hline Advanced Magnetic Materials (university) & produces low-drift, miniature 3 axis magnetometer \\
\hline Delta Doping and APS (JPL) & $\begin{array}{l}\text { combined in advanced solid state detector for solar } \\
\text { wind plasma }\end{array}$ \\
\hline Carbon Velvet (commercial) & $\begin{array}{l}\text { utilized in light trap \& collimator for miniature plasma } \\
\text { sensor }\end{array}$ \\
\hline advanced instrument micro controller (Military) & used as pre-processor \\
\hline
\end{tabular}

\section{ISPI FOR SOLAR PROBE}

With this background about the overall ISPI program we now present the goals for an ISPI for Solar Probe. The capabilities discussed here are consistent with those proposed in response to the NASA NRA 95-15-SS and represent the current state of the system. A complete report will be presented to NASA headquarters at the end of the study phase.

Besides the obvious mass and power savings due to the elimination of functional duplication in ISPI, and the fact that cost and schedule resources can be more efficiently used for the sensor developments, ISPI presents some capability that is particularly advantageous to the solar probe mission. The first of these advantages is ISPI's sensor complement, which include some very impressive capability unique to the science requirements of the mission and without duplication or "bells and whistles" often found in science payloads. The second is the fact that the ISPI architecture allows "interactive" modes where the measurements made by one sensor can be used to affect the operation of another. Third is ISPI's two stage data processing which, when combined with the interactive capability, can be used to improve the science return for the given bit allocation. Much of the study phase for this mission will be devoted to defining the sensor specific and interactive operational requirements that promise the greatest science return for the mission. Additionally, the ISPI testbed for solar probe will receive further definition and ongoing sensor technology studies will refine the sensor configurations.

Table 2 illustrates the current mass and power allocations for the flight configuration and Table 3 shows the ISPI Sensor Specifications as described in the recent proposal.

TABLE 2. ISPI Resource Allocation.

\begin{tabular}{|l|l|l|l|l|}
\hline SENSOR & Mass (kg) & $\begin{array}{l}\text { Average } \\
\text { Power (W) }\end{array}$ & $\begin{array}{l}\text { ISPI output Data in Kbps } \\
\text { Lo res-mode } \quad \text { Hi-res mode }\end{array}$ \\
\hline Plasma & 0.4 & 1.5 & .144 & 36 \\
\hline Magnetometer (2) & 0.05 & 0.1 & .036 & 0.36 \\
\hline Plasma Wave & 0.5 & 0.4 & .48 & 24 \\
\hline Energetic Particles & 0.3 & 0.35 & 0.04 & 1 \\
\hline VIS/EUV imager & 0.8 & 0.8 & 5 & 50 \\
\hline Dust Detector & 0.2 & 0.05 & 0.064 & $<16$ \\
\hline Neutrals & 0.3 & 0.4 & 0.008 & 0.08 \\
\hline coronal imaging & 1.9 & 6.5 & 0.64 & 6.4 \\
\hline \hline ISPI Total & 4.8 & 10.1 & 6.412 & 133.84 \\
\hline
\end{tabular}




\section{SUMMARY}

During the study phase for a Solar Probe ISPI, the science and engineering teams will utilize the architecture and the ISPI testbed to baseline the detailed sytem design for the mission. Likewise, the Co-Investigator teams will be working to refine their high technology sensors, developing the most appropriate measurement capability for Solar Probe. With its ability to perform real-time cooperative science, reduce sensor resource demands and decrease the development burden, we believe ISPI for Solar Probe may serve as an example for how best to devolop other exploratory missions of the future.

TABLE 3. ISPI Sensor Specifications.

\begin{tabular}{|c|c|c|c|c|}
\hline SENSOR & Sensor Technology & Range & Sens. & $\begin{array}{l}\text { Max. Time } \\
\text { Resolution }\end{array}$ \\
\hline Plasma & $\begin{array}{l}\text { Delta-doped Pixelated } \\
\text { SSD; } \\
5 \text { detectors arranged to } \\
\text { cover 2_sr }\end{array}$ & $\begin{array}{l}\text { Protons, electrons: } \\
\mathrm{E}=50 \mathrm{eV}-50 \mathrm{keV} \\
\text { to } \mathrm{AMU}=16: \mathrm{E}=100 \mathrm{eV}-\text { - } \\
100 \mathrm{keV} / \mathrm{n}\end{array}$ & $\begin{array}{l}E=10 \mathrm{eV} \\
\text { for } p^{+}\end{array}$ & $10^{-3} \mathrm{~s}$ \\
\hline Magnetometer & $\begin{array}{l}\text { miniature flux gate ( } 3 \\
\text { axis); } 2 \text { separate sensors }\end{array}$ & $\begin{array}{l} \pm 65536 \mathrm{nT} \text { to } \pm 64 \mathrm{nT} \\
\text { selectable }\end{array}$ & $\begin{array}{l} \pm .5 \mathrm{n} T \text { to } \\
\pm .05 \mathrm{nT}\end{array}$ & $10^{-2} \mathrm{~s}$ \\
\hline Plasma Wave & $\begin{array}{l}\text { B: } 3 \text { axis loop/search coil } \\
\text { E: } 1 \text { axis dipole } \\
\text { Switched capacitor filter } \\
\text { channels }+ \text { wideband } \\
\text { through } 500 \mathrm{~Hz}\end{array}$ & $\begin{array}{l}\text { Freq. Channels: } \\
50 \mathrm{~Hz}-150 \mathrm{kHz} \\
\text { Amplitude: } \\
\text { E: } 10^{-5} \mathrm{v} / \mathrm{m}-10^{1} \mathrm{v} / \mathrm{m} \\
\text { B: } 10^{-2}-10^{3} \mathrm{nT}^{2} / \mathrm{Hz}\end{array}$ & $\begin{array}{l}20 \text { channels } \\
\text { (4/decade, } \\
\text { logarithmic) } \\
5 \%\end{array}$ & $\begin{array}{l}10^{-5} \mathrm{~s} \text { (wave } \\
\text { capture) } \\
1 \mathrm{~s} \text { spectral }\end{array}$ \\
\hline $\begin{array}{l}\text { Energetic } \\
\text { Particles }\end{array}$ & $\begin{array}{l}\text { E vs E SSD telescope } \\
\text { ( } 2 \text { telescopes looking } \\
\text { orthogonally) }\end{array}$ & $\begin{array}{l}\text { Energy: } \\
\mathrm{e}^{-}: 20 \mathrm{keV}--1 \mathrm{MeV} \\
\mathrm{p}^{+}, \mathrm{He}^{+}: 20 \mathrm{keV}-20 \mathrm{MeV} / \mathrm{n} \\
30 \_ \text {FOV each }\end{array}$ & $\begin{array}{l}\text { up to } 10^{4} \\
\text { events/s }\end{array}$ & $\begin{array}{l}10^{1} \mathrm{~s} \text { for } \\
\text { spectrum }\end{array}$ \\
\hline VIS/EUV imager & Frame transfer CCD & Zeeman line magnetograph & TBD & $1 \mathrm{~s}$ \\
\hline Dust detector & $\begin{array}{l}\text { PVDF windows ( } 2) \\
\text { followed by pulse height } \\
\text { analysis }\end{array}$ & $\begin{array}{l}\text { mass: } 10^{-8} \text { to } 10^{-14} \mathrm{~g} \\
\text { flux: }<10^{4} \text { particles } / \mathrm{s}\end{array}$ & $\begin{array}{l}4 \text { logarithmic } \\
\text { channels over } \\
\text { mass range }\end{array}$ & $\begin{array}{l}1 \mathrm{~s} \text { (spectral } \\
\text { mode) }\end{array}$ \\
\hline $\begin{array}{l}\text { Neutrals/ Coronal } \\
\text { Imager }\end{array}$ & $\begin{array}{l}\text { delta-doped SSD for } \\
\text { neutral density and coronal } \\
\text { imaging } \\
\text { ( } 2 \text { sensors) }\end{array}$ & $\begin{array}{l}50 \mathrm{eV} / \mathrm{n}--50 \mathrm{keV} / \mathrm{n} \\
\text { Mass: } 0.3 \mathrm{~kg}\end{array}$ & $E=100 \mathrm{eV}$ & $\begin{array}{l}1 \mathrm{~s} \\
1 \mathrm{~s}\end{array}$ \\
\hline
\end{tabular}

\section{Acknowledgments}

The above work is being sponsored under the following two NASA contracts: NASA NRA-95-12-SL-041 contract \# BP-404-00-73-07-50 "A New Generation Hybrid Advanced Detector;" and NASA NRA-95-15-SS-009 contract \# BP-432-78-00-01-00 "An Integrated Space Physics Instrument (ISPI) for the Near-Sun Flyby Mission." 